\title{
RELATIONS ON SOME SUMMABILITY METHODS
}

\author{
W. T. SULAIMAN
}

(Communicated by Andrew M. Bruckner)

\begin{abstract}
In this paper we prove a new result connecting the summability methods $\left|\bar{N}, p_{n}\right|_{k}$ with either $\left|N, q_{n}\right|_{k}$ or $\left|\bar{N}, w_{n}\right|_{k}$ for given sequences $\left\{p_{n}\right\},\left\{q_{n}\right\}$, and $\left\{w_{n}\right\}$ of positive real constants. Several results, some of them known, are deduced.
\end{abstract}

\section{INTRODUCTION}

Let $\sum a_{n}$ be an infinite series with partial sums $s_{n}$. Let $\sigma_{n}^{\delta}$ and $\eta_{n}^{\delta}$ denote the $n$th Cesàro mean of order $\delta(\delta>-1)$ of the sequences $\left\{s_{n}\right\}$ and $\left\{n a_{n}\right\}$ respectively. The series $\sum a_{n}$ is said to be summable $(C, \delta)$ with index $k$, or simply summable $|C, \delta|_{k}, k \geq 1$, if

$$
\sum_{n=1}^{\infty} n^{k-1}\left|\sigma_{n}^{\delta}-\sigma_{n-1}^{\delta}\right|^{k}<\infty,
$$

or, equivalently,

$$
\sum_{n=1}^{\infty} \frac{1}{n}\left|\eta_{n}^{\delta}\right|^{k}<\infty
$$

Let $\left\{p_{n}\right\}$ be a sequence of real or complex constants with

$$
P_{n}=p_{0}+p_{1}+\cdots+p_{n}, \quad p_{-r}=P_{-r}=0, \quad r=1,2, \ldots
$$

The series $\sum a_{n}$ is said to be summable $\left|N, p_{n}\right|$ if

$$
\sum_{n=1}^{\infty}\left|T_{n}-T_{n-1}\right|<\infty
$$

where

$$
T_{n}=\frac{1}{P_{n}} \sum_{v=0}^{n} p_{n-v} s_{v} \quad\left(T_{-1}=0\right) .
$$

We write $p=\left\{p_{n}\right\}$ and

$$
M=\left\{p: p_{n}>0 \& p_{n+1} / p_{n} \leq p_{n+2} / p_{n+1} \leq 1, n=0,1, \ldots\right\} .
$$

Received by the editors December 4, 1991.

1991 Mathematics Subject Classification. Primary 40G99. 
It is known that for $p \in M(1.1)$ holds if and only if (see [3])

$$
\sum_{n=1}^{\infty} \frac{1}{n P_{n}}\left|\sum_{v=1}^{n} p_{n-v} v a_{v}\right|<\infty .
$$

Definition 1 (Sulaiman [6]). For $p \in M$, we say that $\sum a_{n}$ is summable $\left|N, p_{n}\right|_{k}, k \geq 1$, if

$$
\sum_{n=1}^{\infty} \frac{1}{n}\left|\frac{1}{P_{n}} \sum_{v=1}^{n} p_{n-v} v a_{v}\right|^{k}<\infty .
$$

In the special case in which $p_{n}=A_{n}^{r-1}, r>-1$, where $A_{n}^{r}$ is the coefficient of $x^{n}$ in the power series expansion of $(1-x)^{-r-1}$ for $|x|<1,\left|N, p_{n}\right|_{k}$ summability reduces to $|C, r|_{k}$ summability.

The series $\sum a_{n}$ is said to be summable $\left|\bar{N}, p_{n}\right|_{k}, k \geq 1$, if

$$
\sum_{n=1}^{\infty}\left(\frac{P_{n}}{p_{n}}\right)^{k-1}\left|t_{n}-t_{n-1}\right|^{k}<\infty \quad \text { (Bor [1]) }
$$

where

$$
t_{n}=\frac{1}{P_{n}} \sum_{v=0}^{n} p_{v} s_{v}
$$

If we take $p_{n}=1$, then $\left|\bar{N}, p_{n}\right|_{k}$ summability is equivalent to $|C, 1|_{k}$ summability. In general, these two summabilities are not comparable.

Throughout this paper we set

$$
\begin{gathered}
Q_{n}=q_{0}+q_{1}+\cdots+q_{n}, \quad q_{-1}=Q_{-1}=0 \\
W_{n}=w_{0}+w_{1}+\cdots+w_{n}, \quad w_{-1}=w_{-1}=0 \\
\Delta f_{n}=f_{n}-f_{n+1} .
\end{gathered}
$$

Here we give the following definition:

Definition 2. Let $\left\{p_{n}\right\}$ and $\left\{q_{n}\right\}$ be sequences of positive real constants such that $q \in M$. We say that $\sum a_{n}$ is summable $\left|N, p_{n}, q_{n}\right|_{k}, k \geq 1$, if

$$
\sum_{n=1}^{\infty} \frac{p_{n}}{P_{n} R_{n-1}^{k}}\left|\sum_{v=1}^{n} P_{v-1} q_{n-v} a_{v}\right|^{k}<\infty
$$

where

$$
R_{n}=p_{0} q_{n}+p_{1} q_{n-1}+\cdots+p_{n} q_{0} .
$$

With $t_{n}$ as previously defined,

$$
t_{n}=\frac{1}{P_{n}} \sum_{v=0}^{n} p_{v} \sum_{r=0}^{v} a_{r}=\frac{1}{P_{n}} \sum_{v=0}^{n}\left(P_{n}-P_{v-1}\right) a_{v},
$$

hence

$$
-\Delta t_{n-1}=\frac{p_{n}}{P_{n} P_{n-1}} \sum_{v=1}^{n} P_{v-1} a_{v}
$$


Therefore,

$$
\sum_{n=1}^{\infty}\left(\frac{P_{n}}{p_{n}}\right)^{k-1}\left|\Delta t_{n-1}\right|^{k}=\sum_{n=1}^{\infty} \frac{p_{n}}{P_{n} P_{n-1}^{k}}\left|\sum_{v=1}^{n} P_{v-1} a_{v}\right|^{k}
$$

This shows that $\left|N, p_{n}, 1\right|_{k} \Rightarrow\left|\bar{N}, p_{n}\right|_{k}$.

In 1985 and 1986, respectively, Bor established the following two results.

Theorem A. Let $\left\{p_{n}\right\}$ be a sequence of positive real constants such that as $n \rightarrow$ $\infty$

$$
\text { (i) } n p_{n}=O\left(P_{n}\right), \quad \text { (ii) } P_{n}=O\left(n p_{n}\right) \text {. }
$$

If $\sum a_{n}$ is summable $|C, 1|_{k}$, then it is summable $\left|\bar{N}, p_{n}\right|_{k}, k \geq 1$.

Theorem B. Let $\left\{p_{n}\right\}$ be a sequence of positive real constants such that it satisfies (1.2). If $\sum a_{n}$ is summable $\left|\bar{N}, p_{n}\right|_{k}$, then it is also summable $|C, 1|_{k}$.

\section{We prove}

Theorem 1. Let $\left\{p_{n}\right\},\left\{q_{n}\right\}$, and $\left\{w_{n}\right\}$ be sequences of positive real constants such that $q \in M$ and $\left\{p_{n} / P_{n} R_{n-1}^{k}\right\}$ is nonincreasing for $q_{n} \neq c$. Let $t_{n}$ denote the $\left(\bar{N}, w_{n}\right)$-mean of the series $\sum a_{n}$. Let $\left\{\varepsilon_{n}\right\}$ be a sequence of constants. If

$$
\begin{gathered}
\sum_{n=v+1}^{m+1} \frac{p_{n} q_{n-v-1}}{P_{n} R_{n-1}}=O\left(P_{v}^{-1}\right), \quad m \rightarrow \infty, \\
\sum_{n=1}^{\infty}\left(\frac{P_{n}}{p_{n}}\right)^{k-1}\left|\varepsilon_{n}\right|^{k}\left|\Delta t_{n-1}\right|^{k}<\infty, \\
\sum_{n=1}^{\infty} \frac{p_{n}}{P_{n}}\left(\frac{W_{n}}{w_{n}}\right)^{k}\left|\varepsilon_{n}\right|^{k}\left|\Delta t_{n-1}\right|^{k}<\infty, \\
\sum_{n=1}^{\infty}\left(\frac{P_{n}}{p_{n}}\right)^{k-1}\left(\frac{W_{n}}{w_{n}}\right)^{k}\left|\Delta \varepsilon_{n}\right|^{k}\left|\Delta t_{n-1}\right|^{k}<\infty,
\end{gathered}
$$

and

$$
\sum_{n=1}^{\infty} \frac{p_{n}}{P_{n}}\left(\frac{P_{n-1}}{R_{n-1}}\right)^{k}\left(\frac{W_{n}}{w_{n}}\right)^{k}\left|\varepsilon_{n}\right|^{k}\left|\Delta_{n-1}^{t}\right|^{k}<\infty,
$$

then the series $\sum a_{n} \varepsilon_{n}$ is summable $\left|N, p_{n}, q_{n}\right|_{k}, k \geq 1$.

\section{LEMMAS}

Lemma 1. Let $q \in M$. Then for $0<\gamma \leq 1$,

$$
\sum_{n=v+1}^{\infty} \frac{q_{n-v-1}}{n^{\gamma} Q_{n-1}}=O\left(v^{-\gamma}\right)
$$


Proof. Since $n q_{n}=O\left(Q_{n}\right)$,

$$
\begin{aligned}
\sum_{n=v+1}^{\infty} \frac{q_{n-v-1}}{n^{\gamma} Q_{n-1}} & =\left(\sum_{n=v+1}^{2 v}+\sum_{n=2 v+1}^{\infty}\right) \frac{q_{n-v-1}}{n^{\gamma} Q_{n-1}} \\
& =O\left(\frac{1}{v^{\gamma} Q_{v}}\right) \sum_{n=v+1}^{2 v} q_{n-v-1}+O(1) \sum_{r=v}^{\infty} \frac{q_{r}}{r^{\gamma} Q_{r}} \\
& =O\left(v^{-\gamma}\right)+O(1) \sum_{r=v+1}^{\infty} r^{-\gamma-1}=O\left(v^{-\gamma}\right) .
\end{aligned}
$$

\section{Proof of Theorem 1}

Write

$$
\tau_{n}=\sum_{v=1}^{n} P_{v-1} q_{n-v} a_{v} \varepsilon_{v}
$$

then we have, by Abel's transformation,

$$
\begin{aligned}
& \tau_{n}= \sum_{v=1}^{n} W_{v-1} a_{v}\left(P_{v-1} q_{n-v} \frac{1}{W_{v-1}} \varepsilon_{v}\right) \\
&= \sum_{v=1}^{n-1}\left(\sum_{r=1}^{v} W_{r-1} a_{r}\right) \Delta\left(P_{v-1} q_{n-v} \frac{1}{W_{v-1}} \varepsilon_{v}\right) \\
&+\left(\sum_{r=1}^{n} W_{r-1} a_{r}\right) P_{n-1} q_{0} \frac{1}{W_{n-1}} \varepsilon_{n} \\
&= \sum_{v=1}^{n-1}\left\{-\frac{W_{v-1} W_{v}}{w_{v}} \Delta t_{n-1}\right\} \\
& \times\left\{P_{v-1} \Delta q_{n-v} \frac{1}{W_{v-1}} \varepsilon_{v}+P_{v-1} q_{n-v-1} \frac{w_{v}}{W_{v-1} W_{v}} \varepsilon_{v}\right. \\
&= \sum_{v=1}^{n-1}\left\{-p_{v-1} q_{n-v-1} \frac{1}{W_{v}} \varepsilon_{v}+P_{v} q_{n-v-1} \frac{1}{W_{v}} \Delta \varepsilon_{v}\right\}-P_{n-1} q_{0} \frac{W_{n}}{w_{n}} \varepsilon_{n} \Delta t_{n-1} \\
&\left.\quad \times \varepsilon_{v} \Delta t_{v-1}+P_{v} \Delta t_{v-1}-P_{v-1} q_{n-v-1} \varepsilon_{n-v-1} \Delta t_{v-1}-p_{v-1} q_{n-v-1} \frac{W_{v-1}}{w_{v}} \Delta \varepsilon_{v} \Delta t_{v-1}\right\}-P_{n-1} q_{0} \frac{W_{n}}{w_{n}} \varepsilon_{n} \Delta t_{n-1} \\
&= \tau_{n, 1}+\tau_{n, 2}+\tau_{n, 3}+\tau_{n, 4}+\tau_{n, 5}, \quad \text { say. }
\end{aligned}
$$

In order to prove the theorem, by Minkowski's inequality it is sufficient to show that

$$
\sum_{n=1}^{\infty} \frac{p_{n}}{P_{n} R_{n-1}^{k}}\left|\tau_{n, r}\right|^{k}<\infty, \quad r=1,2,3,4,5
$$


Applying Hölder's inequality,

$$
\begin{aligned}
\sum_{n=1}^{m+1} \frac{p_{n}}{P_{n} R_{n-1}^{k}\left|\tau_{n, 1}\right|^{k}=} & \sum_{n=1}^{m+1} \frac{p_{n}}{P_{n} R_{n-1}^{k}}\left|\sum_{v=1}^{n-1} P_{v-1} \Delta_{v} q_{n-v} \frac{W_{v}}{w_{v}} \varepsilon_{v} \Delta t_{v-1}\right|^{k} \\
\leq & \sum_{n=1}^{m+1} \frac{p_{n}}{P_{n} R_{n-1}^{k}} \sum_{v=1}^{n-1} P_{v-1}^{k}\left|\Delta_{v} q_{n-v}\right|\left(\frac{W_{v}}{w_{v}}\right)^{k}\left|\varepsilon_{v}\right|^{k}\left|\Delta t_{v-1}\right|^{k} \\
& \times\left\{\sum_{v=1}^{n-1}\left|\Delta_{v} q_{n-v}\right|\right\}^{k-1} \\
= & O(1) \sum_{v=1}^{m} P_{v-1}^{k}\left(\frac{W_{v}}{w_{v}}\right)^{k}\left|\varepsilon_{v}\right|^{k}\left|\Delta t_{v-1}\right|^{k} \sum_{n=v}^{m+1} \frac{P_{n}\left|\Delta_{v} q_{n-v}\right|}{P_{n} R_{n-1}^{k}} \\
= & O(1) \sum_{v=1}^{m} \frac{p_{v}}{P_{v}}\left(\frac{P_{v-1}}{R_{v-1}}\right)^{k}\left(\frac{W_{v}}{w_{v}}\right)^{k}\left|\varepsilon_{v}\right|^{k}\left|\Delta t_{v-1}\right|^{k}
\end{aligned}
$$

$$
\begin{aligned}
\sum_{n=2}^{m+1} \frac{p_{n}}{P_{n} R_{n-1}^{k}}\left|\tau_{n, 2}\right|^{k}= & \sum_{n=2}^{m+1} \frac{p_{n}}{P_{n} R_{n-1}^{k}}\left|\sum_{v=1}^{n-1} \frac{P_{v-1}}{p_{v}} p_{v} q_{n-v-1} \varepsilon_{v} \Delta t_{v-1}\right|^{k} \\
& \leq \sum_{n=2}^{m+1} \frac{p_{n}}{P_{n} R_{n-1}} \sum_{v=1}^{n-1}\left(\frac{P_{v-1}}{p_{v}}\right)^{k} p_{v} q_{n-v-1}\left|\varepsilon_{v}\right|^{k}\left|\Delta t_{v-1}\right|^{k} \\
& \times\left\{\sum_{v=1}^{n-1} \frac{p_{v} q_{n-v-1}}{R_{n-1}}\right\}^{k-1} \\
= & O(1) \sum_{v=1}^{m}\left(\frac{P_{v}}{p_{v}}\right)^{k} p_{v}\left|\varepsilon_{v}\right|^{k}\left|\Delta t_{v-1}\right|^{k} \sum_{n=v+1}^{m+1} \frac{p_{n} q_{n-v-1}}{P_{n} R_{n-1}} \\
= & O(1) \sum_{v=1}^{m}\left(\frac{P_{v}}{p_{v}}\right)^{k-1}\left|\varepsilon_{v}\right|^{k}\left|\Delta t_{v-1}\right|^{k},
\end{aligned}
$$

$$
\begin{aligned}
\sum_{n=2}^{m+1} \frac{p_{n}}{P_{n} R_{n-1}^{k}}\left|\tau_{n, 3}\right|^{k}= & \sum_{n=2}^{m+1} \frac{p_{n}}{P_{n} R_{n-1}^{k}}\left|\sum_{v=1}^{n-1} p_{v} q_{n-v-1} \frac{W_{v-1}}{w_{v}} \varepsilon_{v} \Delta t_{v-1}\right|^{k} \\
\leq & \sum_{n=2}^{m+1} \frac{p_{n}}{P_{n} R_{n-1}} \sum_{v=1}^{n-1} p_{v} q_{n-v-1}\left(\frac{W_{v-1}}{w_{v}}\right)^{k}\left|\varepsilon_{v}\right|^{k}\left|\Delta t_{v-1}\right|^{k} \\
& \times\left\{\sum_{v=1}^{n-1} \frac{p_{v} q_{n-v-1}}{R_{n-1}}\right\}^{k-1} \\
= & O(1) \sum_{v=1}^{m} p_{v}\left(\frac{W_{v}}{w_{v}}\right)^{k}\left|\varepsilon_{v}\right|^{k}\left|\Delta t_{v-1}\right|^{k} \sum_{n=v+1}^{m+1} \frac{p_{n} q_{n-v-1}}{P_{n} R_{n-1}} \\
= & O(1) \sum_{v=1}^{m} \frac{p_{v}}{P_{v}}\left(\frac{W_{v}}{w_{v}}\right)^{k}\left|\varepsilon_{v}\right|^{k}\left|\Delta t_{v-1}\right|^{k},
\end{aligned}
$$




$$
\begin{aligned}
& \sum_{n=2}^{m+1} \frac{p_{n}}{P_{n} R_{n-1}^{k}\left|\tau_{n, 4}\right|^{k}=} \sum_{n=2}^{m+1} \frac{p_{n}}{P_{n} R_{n-1}^{k}}\left|\sum_{v=1}^{n-1} P_{v} q_{n-v-1} \frac{W_{v-1}}{w_{v}} \Delta \varepsilon_{v} \Delta t_{v-1}\right|^{k} \\
& \leq \sum_{n=2}^{m+1} \frac{p_{n}}{P_{n} R_{n-1}} \sum_{v=1}^{n-1}\left(\frac{P_{v}}{p_{v}}\right)^{k} p_{v} q_{n-v-1}\left(\frac{W_{v-1}}{w_{v}}\right)^{k}\left|\Delta \varepsilon_{v}\right|^{k}\left|\Delta t_{v-1}\right|^{k} \\
& \times\left\{\sum_{v=1}^{n-1} \frac{p_{v} q_{n-v-1}}{R_{n-1}}\right\}^{k-1} \\
&= O(1) \sum_{v=1}^{m}\left(\frac{P_{v}}{p_{v}}\right)^{k} p_{v}\left(\frac{W_{v}}{w_{v}}\right)^{k}\left|\Delta \varepsilon_{v}\right|^{k}\left|\Delta t_{v-1}\right|^{k} \sum_{n=v+1}^{m+1} \frac{p_{n} q_{n-v-1}}{P_{n} R_{n-1}} \\
&= O(1) \sum_{v=1}^{m}\left(\frac{P_{v}}{p_{v}}\right)^{k-1}\left(\frac{W_{v}}{w_{v}}\right)^{k}\left|\Delta \varepsilon_{v}\right|^{k}\left|\Delta t_{v-1}\right|^{k}, \\
& \sum_{n=1}^{m} \frac{p_{n}}{P_{n} R_{n-1}^{k}\left|\tau_{n, 5}\right|^{k}}=\sum_{n=1}^{m} \frac{p_{n}}{P_{n} R_{n-1}^{k}}\left|P_{n-1} q_{0} \frac{W_{n}}{w_{n}} \varepsilon_{n} \Delta t_{n-1}\right|^{k} \\
&=O(1) \sum_{n=1}^{m} \frac{p_{n}}{P_{n}}\left(\frac{P_{n-1}}{R_{n-1}}\right)^{k}\left(\frac{W_{n}}{w_{n}}\right)^{k}\left|\varepsilon_{n}\right|^{k}\left|\Delta t_{n-1}\right|^{k} .
\end{aligned}
$$

\section{Applications}

Corollary 1 (Bor and Thorpe [3]). Let $\left\{p_{n}\right\}$ and $\left\{w_{n}\right\}$ be sequences of positive real constants. If

$$
\text { (i) } p_{n} W_{n}=O\left(P_{n} w_{n}\right), \quad \text { (ii) } P_{n} w_{n}=O\left(p_{n} W_{n}\right),
$$

then the series $\sum a_{n}$ is summable $\left|\bar{N}, p_{n}\right|_{k}$ whenever it is summable $\left|\bar{N}, w_{n}\right|_{k}$, $k \geq 1$.

Proof. The proof follows from Theorem 1 by putting $\varepsilon_{n}=1, q_{n}=1$.

Corollary 2 (Theorems A and B). If (1.2) is satisfied, then the series $\sum a_{n}$ is summable $|C, 1|_{k}$ if and only if it is summable $\left|\bar{N}, p_{n}\right|_{k}, k \geq 1$.

Proof. $(\Rightarrow)$ follows from Corollary 1 by putting $p_{n}=1$.

$(\Leftarrow)$ follows from Corollary 1 by putting $q_{n}=1$.

\section{ACKNOWLEDGMENTS}

I am very grateful to the referee for his interesting comments.

\section{REFERENCES}

1. H. Bor, On two summability methods, Math. Proc. Cambridge Philos. Soc. 97 (1985), 147149.

2. __ A note on two summability methods, Proc. Amer. Math. Soc. 98 (1986), 81-84.

3. H. Bor and B. Thorpe, On some absolute summability methods, Analysis 7 (1987), 145-152.

4. G. Das, Tauberian theorems for absolute Nörlund summability, Proc. London. Math. Soc. (3) 19 (1969), 357-384. 
5. M. A. Sarigöl, Necessary and sufficient conditions for the equivalence of the summability methods $\left|\bar{N}, P_{n}\right|_{k}$ and $|C, 1|_{k}$, Indian J. Pure Appl. Math. 22 (1991), 483-489.

6. W. T. Sulaiman, Notes on two summability methods, Pure Appl. Math. Sci. 31 (1990), 59-68.

Department of Mathematics, Mosul University, Mosul, IraQ 